\title{
Attitude Survey of Young People to Regional Resource Sharing with Quantification Method Type II
}

\author{
Shimpei Matsumoto *, Nobuyuki Ohigashi *
}

\begin{abstract}
To support the daily life of vulnerable road users, mainly older people who are living in suburban slope residential estates without enough public transport service, we have shown the concept of regional resource sharing. Besides, based on the concept, we have developed a web service, Mutual Assistance Support System called MASS. The role of MASS is to facilitate the encounter between local community people, to provide the opportunity of resource sharing, and to solve the difficulties in daily life. The active participation of young people would be essential to make MASS more effective for vulnerable road users because young people will provide most of the resources, such as skills similar to other skill-sharing services. Therefore, in this paper, in order to examine the sustainability of MASS, we conducted an attitude survey on young people's awareness about their resource sharing, including skills at their local community, and analyzed it with Quantification Method Type II. The analysis result unveiled a rough trend of the youth consciousness about resource sharing and the local community. Still, the analysis result also showed the limitation of Quantification Method Type II.
\end{abstract}

Keywords: Vulnerable road users, ride-sharing, mutual assistance, local community activation, Quantification Method Type II.

\section{Introduction}

In Hiroshima City, the central city of Hiroshima prefecture in Japan, there are many slope residential estates at its surrounding mountains. These estates had been developed under the theory of neighborhood unit [1]. Still, the living environment in these areas is not suitable for vulnerable road users, mainly older people, due to various reasons such as being on a slope and away from the central. Therefore, we have examined a new public transportation means which keeps the service level of these estates for the longest possible time.

To embody the form of public transportation means, we had first investigated the residents' traffic awareness at the suburban slope residential areas around Itsukaichi district, the main bedroom community of Hiroshima City [2]. This survey unveiled that municipal public transportation services are not enough at all for the vulnerable road users. Then, based

\footnotetext{
* Hiroshima Institute of Technology, Hiroshima, Japan
} 
on the result, we had shown the concept of a transportation support model for vulnerable road users living in the suburban slope residential areas without enough public transportation means. We have also designed and developed a prototype of Web service, Mutual Assistance Support System called MASS [3][4]. MASS can provide efficient transportation means for both vulnerable road users and municipality by utilizing resident's help for supporting their daily trips within a short distance. MASS can realize various kinds of mutual assistance. For example, a resident who will move to his/her destination by private car can meet another resident's needs at the same time as his/her original purpose. Besides, we had improved MASS to a resource-sharing service to support vulnerable road user's daily life [5][6][7] along with the concept of sharing economy [8][9].

To enhance the resources provided through MASS, active participation by young people is indispensable because young people will provide most of the resources, such as skills similar to other skill-sharing services. Many of skill-sharing services have been successful, thanks to the active use of youth [10][11][12][13]. Therefore, in this paper, in order to examine the sustainability of MASS, we focused on the attitude survey result on young people's awareness about their resource sharing, including skills at their local community, and analyzed it with Quantification Method Type II. The analysis result unveiled a rough trend of the youth consciousness about resource sharing and the local community, which have not been clarified so far. Note that the contribution of this paper is to consider the previously reported findings [14] in more detail.

\section{Background}

\subsection{Current Situation in the Suburban Slope Residential Areas}

Itsukaichi District, the main bedroom suburb of Hiroshima City and the target region of this study, has some housing estates systematically developed. Many of these housing estates are far from the central area and also located at the slope area. Besides, the only public transportation that is readily available in these residents' daily lives is the regular bus, so they seem to be unsuitable for vulnerable road users, mainly older people who cannot freely move.

Based on the above background, In 2009, we had investigated the actual situation with the questionnaire for 2951 households [2] to clarify the degree of satisfaction and its reason for current public transportation means, which is daily used for a short-distance trip like shopping. First, the questionnaire result unveiled the current situation that many residents were strongly dependent on their private car due to the insufficiency of public transportation services near their living areas. Specifically, they indicated that public transport services were not adequate because of the lack of a station near their homes and the low number of bus services. The questionnaire also showed the difficulty of daily use of a private vehicle for older people. Since their residential areas are far from the city area, older people did not have enough confidence to drive safely. The older people desired to use public transportation if possible, but they were forced to use their cars because of the dissatisfaction with the current public transportation service. As one of the main reasons for dissatisfaction, there were no bus routes within reasonable walking distance in some areas. To reduce their frustration about public transportation, many older people desired the improvement of public bus service. Especially, their request was not to enhance the existing bus service but to introduce a convenient and flexible transportation service like a demand-responsive bus or a community bus. 
After considering the questionnaire result and also some previous works such as [15], we had examined a community bus-based new public transportation means. As a result, we found introducing a public transportation service was not realistic because such areas have only a few people and then extremely unprofitable. In fact, some local governments operate community bus traveling throughout the residential area and main commercial places, including bus stops, shopping stores, and rail stations. Still, most of them have not been monetized. Then, we had had to examine a realistic plan to improve the service level of transportation, even in these depopulated areas. Eventually, we concluded that the only way of the improvement is to utilize mutual aid between residents.

\subsection{Previous Studies}

Personal/mini transportation means for a local community is often referred to as DRT (Demand Responsive Transport) or FTS (Flexible Transport Services) [16][17]. The contributions of most of the studies on DRT/FTS are the provision of new knowledge on the design methodology of transportation service based on individual transportation means [18]. For example, Kawabata et al. revealed that walking distance from one's home to a bus stop is a significant barrier to the use of a bus for older people [19]. Some studies have reported the simulation models introducing a transportation service for local city and its neighboring parts [20][21]. Kleiner et al. presented a multi-agent dynamic ride-sharing model [22], allowing to trade off the minimization of vehicle kilometers traveled with the overall probability of successful ride-shares. Agatz et al. showed the optimization challenges that arise when developing a technology to support ride-sharing from the viewpoint of operations research [23]. Some studies showed methods/concepts for reasonably realizing DRT / FTS. Yotsutsuji et al. showed a frame of volunteer-based dynamic ride-sharing to improve the transportation of the local community [24]. They analyzed the feasibility of ride-sharing by pre-survey data and examined its availability through a field experiment. Their effort seems to be similar to our approach, but the main contribution of our studies is different in terms of the point that we have been aiming to construct a structure available for supporting vulnerable road users at the suburban residential areas [3]. Yotsutsuji's and our approach are considered to fall under the frame of "Town development company" [25], which is the concept aimed at maintaining and improving public services through the mutual aid of local residents.

\section{Mutual Assistance Support System}

The core service of MASS is to encourage people to meet each other in their local region. MASS facilitates the encounter by personal information sharing, such as each one's skills and troubles, and aims to solve a troubled resident's problem in daily life [5][6][7][26]. MASS provides primary two services: the one is the time-dependent skill/trouble information sharing to coordinate someone's request and acceptance, and the other is the communication support to negotiate the condition of mutual assistance. Most troubles might be on a daily short-distance travel, but the requests are not limited to these and include such as gardening, dog-walking, and maintenance of household appliances. For example, with MASS, a troubled person, who has no daily transportation means but needs to get out, can find a person with easy procedures, who lives near the troubled person and ask to bring out together. Although such mutual aid is a volunteer activity, a helper can obtain decent wages as a donation, and MASS securely manages all transactions of the charges between users. 
Here is the flow of resource sharing. First, a troubled resident called as "client" posts a request to MASS. For example, a resident, who needs to go to a hospital but does not have transportation means or needs someone's help on the way to the hospital, can send a request with text message or voice chat. Second, if a user called as "server" who readies to help another decides to accept the request after checking the details of another resident's request such as conditions of schedule and personal information, MASS connects the server and the client by the direct message (chat) function. These users can negotiate at the communication function and aim to reach an agreement. In this flow, MASS will promote the rationalization and the efficiency of transports on people and goods. Eventually, since MASS encourages the meetings of each resident, the authors expect that MASS can help reproduce a local community.

\subsection{Condition of Attitude Survey and the Summary of Its Analysis Result}

Many of skill-sharing services have been successful, thanks to the active use of youth. Thus, to enhance the resources provided through MASS, young people's active participation would be indispensable because young people will provide most of the resources. In order to examine the sustainability of MASS, we conducted an attitude survey on young people's awareness of sharing their resources in their local community. We employed 88 Hiroshima residents from 20 years old to 24 years old as examinees and obtained the awareness data by a questionnaire. Each response of the questionnaire was on the 4-grade Likert scale (strongly agree, weakly agree, weakly disagree, strongly disagree); 4 is the maximum (positive), and 1 is the minimum (negative). First, examinees listened to about 10 minutes of presentation on MASS. Then they utilized the prototype of MASS freely with sufficient time while receiving its explanation to operate with proper procedures from an experimenter. After that, each examinee answered his/her opinion for each item in the questionnaire.

First of all, we confirmed whether the examinees sufficiently understood the concept of MASS to check the reliability of the responses. As a result, we confirmed that all reactions are reliable to analyze.

First, we asked examinees for their subjective opinion on MASS by following two questions:

- $\mathrm{Q}_{1}$ : When MASS is actually launched, would you like to utilize it as a user to provide your resource?

- $\mathrm{Q}_{2}$ : When MASS is actually launched, would you like to utilize it as a user to get another one's resource?

Next, we also gave the examinees three questions about whether MASS will be utilized in society, which asked their views from an objective standpoint, not from their subjectivity.

- $\mathrm{Q}_{3}$ : Do you think that local residents will use MASS to share resources to support one's daily life, such as housework, gardening, maintenance of household appliances, repairing an item of furniture, and pet care? Give your opinion from an objective standpoint.

- $\mathrm{Q}_{4}$ : Do you think that local residents will use MASS to share resources on transportation such as the assistance of shopping, and short-distance ride-sharing for commuting or shopping? Give your opinion from an objective standpoint.

- $\mathrm{Q}_{5}$ : Do you think providing services by local people's mutual aid instead of public services ideal? 
Table 1: The detail of accuracy when $\mathrm{Q}_{1}$ is the objective variable

\begin{tabular}{c|ccc}
\hline & \multicolumn{3}{|c}{ Discrimination } \\
Observation & 1 & 0 & Accuracy \\
\hline 1 & 46 & 0 & $100.00 \%$ \\
0 & 3 & 39 & $92.86 \%$ \\
\hline
\end{tabular}

$\mathrm{Q}_{1}$ and $\mathrm{Q}_{2}$ are evaluations as to whether the examinees wanted to use MASS. $\mathrm{Q}_{3}$ and $\mathrm{Q}_{4}$ are evaluations as to whether the examinees thought MASS good as a general business from an objective standpoint. $\mathrm{Q}_{5}$ is the opinion for utilizing mutual assistance to improve public service.

\subsection{Basic Analysis with Quantification Method Type II}

We examined the reasons for the response of $\mathrm{Q}_{1}$ by multiple selection methods. Positive reasons $\left(\mathrm{R}_{1}-\mathrm{R}_{4}\right)$ and negative reasons $\left(\mathrm{R}_{5}-\mathrm{R}_{8}\right)$ are as follows.

- $\mathrm{R}_{1}$ : I want to get rewards.

- $\mathrm{R}_{2}$ : I want to make a new connection with people in the local community.

- $\mathrm{R}_{3}$ : I am interested in a new service.

- $\mathrm{R}_{4}$ : I want to contribute to the local community.

- $\mathrm{R}_{5}$ : I am not interested in making money in such this way.

- $\mathrm{R}_{6}$ : I am nervous (scared) to engage with unknown others.

- $\mathrm{R}_{7}$ : I do not want to disclose personal information.

- $\mathrm{R}_{8}$ : I do not go out much.

Similarly, we got the reasons for the response of $\mathrm{Q}_{2}$ by multiple selection methods. Positive reasons (abbreviated as $R_{9}-R_{11}$ ) and negative reasons (abbreviated as $R_{12}-R_{15}$ ) are as follows.

- $\mathrm{R}_{9}$ : I feel MASS is useful.

- $\mathrm{R}_{10}$ : I want to join the interaction with local people.

- $\mathrm{R}_{11}$ : I want to reduce waste/to save money.

- $\mathrm{R}_{12}$ : I do not need another's help because I will do my own thing myself.

- $\mathrm{R}_{13}$ : I am worried about some kinds of accidents/troubles.

- $\mathrm{R}_{14}$ : I feel MASS is useful, but it is uneasy to interact with unknown people.

- $\mathrm{R}_{15}$ : I am not interested in the local community.

We applied Quantification Method Type II to clarify the relationship between the evaluation of $\mathrm{Q}_{1} / \mathrm{Q}_{2}$ and their reasons $\mathrm{R}_{1}-\mathrm{R}_{15}$. First, the responses of $\mathrm{Q}_{1}$ and $\mathrm{Q}_{2}$ were binarized where 3 and 4 were transformed into 1 , and 1 and 2 were transformed into 0 . Next, we applied Quantification Method Type II to two kinds of input data: one of whose the objective variable is $Q_{1}$ and the explanatory variables are $R_{1}-R_{8}$, and the other of whose the objective variable is $\mathrm{Q}_{2}$ and the explanatory variables are $\mathrm{R}_{9}-\mathrm{R}_{15}$.

First, the objective variables were discriminated against by using constructed models. The discriminating accuracy of the model $\mathrm{Q}_{1}$ was $96.59 \%$ (see Table 1), and the model $\mathrm{Q}_{2}$ was $100 \%$. Therefore, the relationship between $\mathrm{Q}_{1} / \mathrm{Q}_{2}$ and their reasons would be strong, and we can consider that each examinee's expectation for MASS strongly depends on the reasons $R_{1}-R_{15}$ or is summarized by these reasons. 
Table 2: Category scores of each explanatory variable when $\mathrm{Q}_{1}$ is the objective variable

\begin{tabular}{c|cccccccc}
\hline Items & $\mathrm{R}_{1}$ & $\mathrm{R}_{2}$ & $\mathrm{R}_{3}$ & $\mathrm{R}_{4}$ & $\mathrm{R}_{5}$ & $\mathrm{R}_{6}$ & $\mathrm{R}_{7}$ & $\mathrm{R}_{8}$ \\
\hline C.S.(=0) & -0.15 & -0.13 & -0.06 & -0.04 & 0.09 & 0.45 & -0.04 & 0.04 \\
C.S.(=1) & 0.46 & 0.32 & 0.19 & 0.16 & -0.69 & -0.69 & 0.27 & -0.28 \\
\hline
\end{tabular}

Table 3: Category scores of each explanatory variable when $\mathrm{Q}_{2}$ is the objective variable

\begin{tabular}{c|ccccccc}
\hline Items & $\mathrm{R}_{9}$ & $\mathrm{R}_{10}$ & $\mathrm{R}_{11}$ & $\mathrm{R}_{12}$ & $\mathrm{R}_{13}$ & $\mathrm{R}_{14}$ & $\mathrm{R}_{15}$ \\
\hline C.S. $(=0)$ & -0.51 & -0.07 & -0.08 & 0.08 & 0.14 & 0.05 & 0.02 \\
C.S. $(=1)$ & 0.71 & 0.54 & 0.56 & -0.27 & -0.28 & -0.16 & -0.28 \\
\hline
\end{tabular}

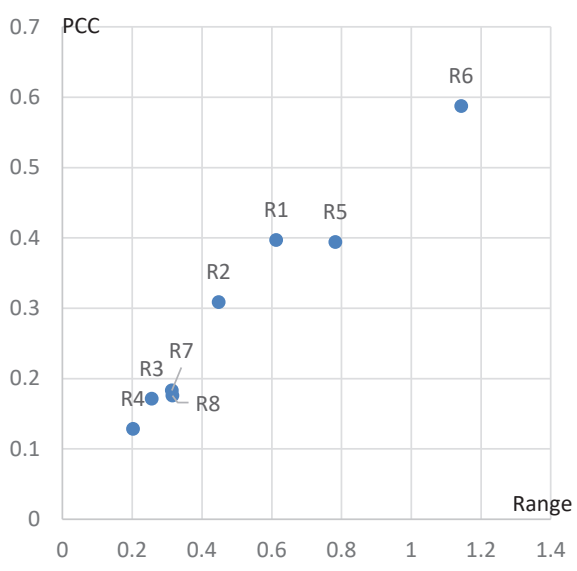

(a) The Model of $Q_{1}$

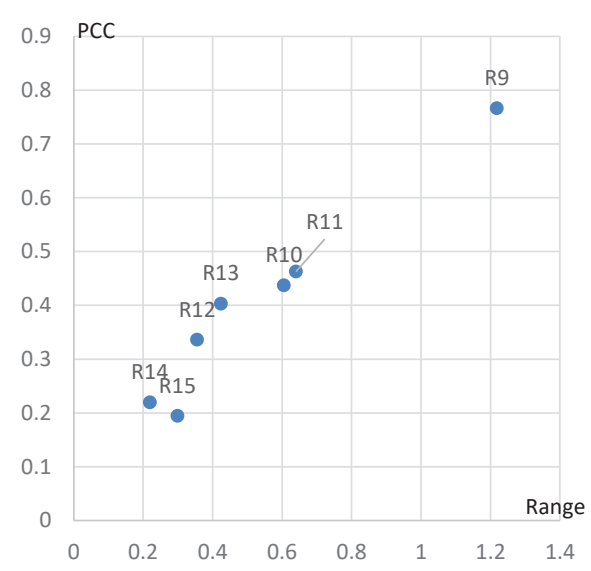

(b) The Model of $\mathrm{Q}_{2}$

Figure 1: Partial correlation coefficients (PCC) and Range in two models of $\mathrm{Q}_{1}$ and $\mathrm{Q}_{2}$

Table 2 and Table 3 show the category scores of each explanatory variable. Similarly, Figure 1 shows the partial correlation coefficients and ranges of each explanatory variable. Here, the category score is written as C.S., the partial correlation coefficient is written as PCC, and other tables also follow this description. Also, the range denotes the difference between the highest categorical score and the lowest categorical score within the explanatory variable. The larger the range, the greater the value of that explanatory variable is in the discriminating.

From Table 2, we can see the maximum range of $\mathrm{Q}_{1}$ at $\mathrm{R}_{6}$. Figure 1 also shows a high value of $R_{6}$. The result might suggest that there is strong resistance to engaging with unknown people, even the local area familiar with the examinees. Perhaps, local young people have little experience cooperating with local people, so they do not trust other people. This analysis result might be a piece of remarkable evidence suggesting that some of the young people are independent of their area.

Next in Table 3, the maximum range of $\mathrm{Q}_{2}$ was $\mathrm{R}_{9}$. Figure 1 also shows a high value of $R_{9}$. These results suggest that young people have a low resistance to using a resourcesharing service if they feel it convenient. With the spread of Internet services, it is becoming common to interact with various people beyond usual relations. Some of the examinees may have a lot of experiences for using a service to interact with strangers, such as Internet-based online auction and free-market services. Therefore, they may feel MASS close, similar 
to such Internet-based resource-sharing services. Therefore, we can consider that young people will actively use MASS as long as MASS has a mechanism in place that they find convenient. At the same time, if attractive resources for young people are provided enough, we can expect to see a revitalization of exchange among residents and the spread of MASS. However, it is most important to have sufficient resources first. Especially, more than youth participation as service users, participation as service providers is essential for solving the issue of vulnerable road users. As shown in Table 2, setting appropriate rewards is important as a motivation for providing the user's resources. Therefore, we could obtain a hypothesis that MASS has to set attractive rewards for young people for its activation from these results until the MASS penetrates the region.

\subsection{Advanced Analysis with Quantification Method Type II}

Same with the analysis of $\mathrm{Q}_{1}$ and $\mathrm{Q}_{2}$, we applied Quantification Method Type II to construct models for $\mathrm{Q}_{3}-\mathrm{Q}_{5}$ after the response values of $\mathrm{Q}_{3}-\mathrm{Q}_{5}$ were binarized. The criteria for binarization were the same as for the models of $\mathrm{Q}_{1}$ and $\mathrm{Q}_{2}$. We constructed three models whose objective variable is $\mathrm{Q}_{3} / \mathrm{Q}_{4} / \mathrm{Q}_{5}$, and explanatory variables are $\mathrm{R}_{1}-\mathrm{R}_{15}$. Before constructing these models, we have to show the validity of giving all of $R_{1}-R_{15}$ to the model as explanatory variables. For this point, we first constructed two models with $\mathrm{Q}_{1} / \mathrm{Q}_{2}$ as the objective variable and $R_{1}-R_{15}$ as explanatory variables. Then, we compared the discriminating accuracy between this model and the result of the previous section. As a result of analyzing based on this idea, the gaps in the discriminating accuracy were small. Concretely, we obtained a model with the discriminating accuracy $95.45 \%$ when $\mathrm{Q}_{1}$ was set as the objective variable, and $R_{1}-R_{15}$ were set as the explanatory variables. Similarly, the discriminating accuracy of the model whose objective variable is $\mathrm{Q}_{2}$, and the explanatory variables are $R_{1}-R_{15}$ was $100.0 \%$. Based on the results, we considered that there is no problem giving all of $\mathrm{R}_{1}-\mathrm{R}_{15}$ as explanatory variables.

Table 4 shows the accuracy of the model with $\mathrm{Q}_{3}$ as the objective variable, Table 5 shows its category scores and Figure 2 (a) shows partial correlation coefficients and ranges of explanatory variables. We can see the low accuracy of this model from Table $4 . R_{1}-R_{15}$ means the examinee's subjective impression about MASS. $\mathrm{Q}_{3}$ represents the examinee's objective view about the popularization of MASS. Thus, these tables' results suggest that the relationship between each examinee's subjective and objective evaluations was not strong. This result indicates that a certain number of examinees thought MASS would be popular in the local area even if they did not want to use it. Alternatively, the result also suggests its vice versa. The examinees' evaluation of Q3 might depend on the environment in which they were born and raised. Therefore, this result suggests that there are certainly places with residents who need MASS. Also, this result provides an assumption that disseminating MASS from the areas is effective.

Likewise, Table 6 shows the discriminating accuracy of the model $\mathrm{Q}_{4}$, Table 7 shows its category scores, and Figure 2 (b) shows partial correlation coefficients and ranges of explanatory variables. The discriminating accuracy of the model $\mathrm{Q}_{4}$ was low same with the model $\mathrm{Q}_{3}$. But, the discriminating accuracy of the model of $\mathrm{Q}_{4}$ was slightly higher than that of the model of $\mathrm{Q}_{3}$. This result suggests that residents' perceptions of resource sharing with respect to transportation are closer to the views of young people than to various kinds of resources. While there was an approximate agreement in the discrimination accuracy of the models of $\mathrm{Q}_{3}$ and $\mathrm{Q}_{4}$, these category scores were different. For the model of $\mathrm{Q}_{3}$, we focus on three factors $R_{3}, R_{7}$, and $R_{8}$ (see Figure 2 (a)) that are particularly strong. From the 
Table 4: The detail of accuracy when $\mathrm{Q}_{3}$ is the objective variable (Accuracy: $73.86 \%$ )

\begin{tabular}{c|ccc}
\hline & \multicolumn{3}{|c}{ Discrimination } \\
Observation & 1 & 0 & Accuracy \\
\hline 1 & 40 & 12 & $76.92 \%$ \\
0 & 11 & 25 & $69.44 \%$ \\
\hline
\end{tabular}

Table 5: Category scores of each explanatory variable when $\mathrm{Q}_{3}$ is the objective variable

\begin{tabular}{c|cccccccc}
\hline Items & $\mathrm{R}_{1}$ & $\mathrm{R}_{2}$ & $\mathrm{R}_{3}$ & $\mathrm{R}_{4}$ & $\mathrm{R}_{5}$ & $\mathrm{R}_{6}$ & $\mathrm{R}_{7}$ & $\mathrm{R}_{8}$ \\
\hline C.S.(=0) & -0.08 & -0.15 & -0.18 & -0.07 & -0.01 & 0.06 & 0.09 & 0.10 \\
C.S.(=1) & 0.23 & 0.36 & 0.53 & 0.24 & 0.07 & -0.09 & -0.61 & -0.75 \\
\hline Items & $\mathrm{R}_{9}$ & $\mathrm{R}_{10}$ & $\mathrm{R}_{11}$ & $\mathrm{R}_{12}$ & $\mathrm{R}_{13}$ & $\mathrm{R}_{14}$ & $\mathrm{R}_{15}$ & \\
\hline C.S.(=0) & -0.13 & 0.05 & 0.00 & 0.08 & 0.12 & 0.04 & 0.01 & \\
C.S.(=1) & 0.18 & -0.35 & 0.02 & -0.26 & -0.24 & -0.11 & -0.07 & \\
\hline
\end{tabular}

Table 6: The detail of accuracy when $\mathrm{Q}_{4}$ is the objective variable (Accuracy: 79.55\%)

\begin{tabular}{c|ccc}
\hline & \multicolumn{3}{|c}{ Discrimination } \\
Observation & 1 & 0 & Accuracy \\
\hline 1 & 48 & 11 & $81.36 \%$ \\
0 & 7 & 22 & $75.86 \%$ \\
\hline
\end{tabular}

Table 7: Category scores of each explanatory variable when $\mathrm{Q}_{4}$ is the objective variable

\begin{tabular}{c|cccccccc}
\hline Items & $\mathrm{R}_{1}$ & $\mathrm{R}_{2}$ & $\mathrm{R}_{3}$ & $\mathrm{R}_{4}$ & $\mathrm{R}_{5}$ & $\mathrm{R}_{6}$ & $\mathrm{R}_{7}$ & $\mathrm{R}_{8}$ \\
\hline C.S.(=0) & 0.01 & 0.07 & 0.23 & 0.02 & -0.17 & -0.23 & 0.00 & -0.03 \\
C.S. $(=1)$ & -0.03 & -0.18 & -0.68 & -0.09 & 1.32 & 0.35 & -0.02 & 0.21 \\
\hline Items & $\mathrm{R}_{9}$ & $\mathrm{R}_{10}$ & $\mathrm{R}_{11}$ & $\mathrm{R}_{12}$ & $\mathrm{R}_{13}$ & $\mathrm{R}_{14}$ & $\mathrm{R}_{15}$ & \\
\hline C.S.(=0) & 0.40 & -0.05 & -0.02 & 0.06 & -0.08 & 0.18 & 0.05 & \\
C.S. $(=1)$ & -0.55 & 0.37 & 0.16 & -0.19 & 0.15 & -0.55 & -0.67 & \\
\hline
\end{tabular}

values of these category scores, we can understand the strength "interest in a new service" $\left(\mathrm{R}_{3}\right)$ as the positive factor and "disclosing personal information" $\left(\mathrm{R}_{7}\right)$ and "going out" $\left(\mathrm{R}_{8}\right)$ as the negative factors in the model of $\mathrm{Q}_{3}$. This result is quite natural, and it might be evidence that the examinees felt that local residents would be interested in MASS. It may seem unnatural that the signs of category scores in $R_{1}-R_{4}, R_{9}, R_{10}$, and $R_{11}$ are not aligned (see Table 5). However, since these PCCs and Ranges are not large (see Figure 2), it can be said that there is no need to worry about them.

The category scores in $\mathrm{Q}_{4}$, as shown in Table 7 is difficult to interpret at first glance. However, since this result is only a sign reversal, we can see a reasonable trend if all signs are interpreted in reverse. we focus on three factors $R_{3}, R_{5}$, and $R_{9}$ (see Figure 2 (b)). The strong value of $R_{3}$ is common to the model of $Q_{3}$ and $Q_{4}$. On the other hand, $R_{5}$ and $R_{9}$ are different from $Q_{3}$. Since $R_{9}$ is a clear reason for "feeling it useful," $\left(R_{9}\right)$ the strength of the factor is easily convincing. But, the strength of the $R_{5}$ is surprising, and the interpretation is not easy. It may suggest the need to have a response in place when some kind of trouble arises, rather than a reward. Especially, sharing resources on transportation has 


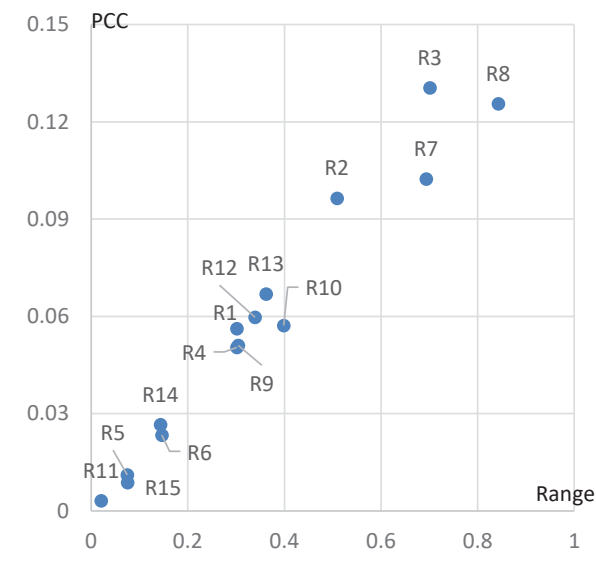

(a) The Model of $Q_{3}$

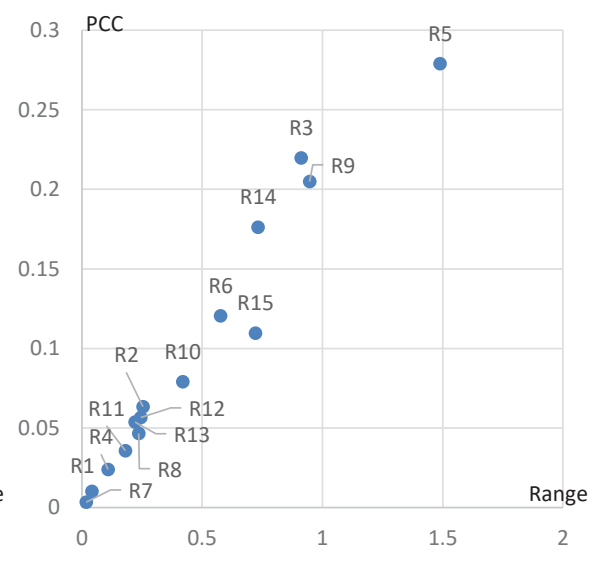

(b) The Model of $\mathrm{Q}_{4}$

Figure 2: Partial correlation coefficients (PCC) and Range in two models of $\mathrm{Q}_{3}$ and $\mathrm{Q}_{4}$

Table 8: The detail of accuracy when $\mathrm{Q}_{5}$ is the objective variable (Accuracy: 75.00\%)

\begin{tabular}{c|ccc}
\hline & \multicolumn{3}{|c}{ Discrimination } \\
Observation & 1 & 0 & Accuracy \\
\hline 1 & 55 & 19 & $74.32 \%$ \\
0 & 3 & 11 & $78.57 \%$ \\
\hline
\end{tabular}

Table 9: Category scores of each explanatory variable when $\mathrm{Q}_{5}$ is the objective variable

\begin{tabular}{c|cccccccc}
\hline Items & $\mathrm{R}_{1}$ & $\mathrm{R}_{2}$ & $\mathrm{R}_{3}$ & $\mathrm{R}_{4}$ & $\mathrm{R}_{5}$ & $\mathrm{R}_{6}$ & $\mathrm{R}_{7}$ & $\mathrm{R}_{8}$ \\
\hline C.S. $(=0)$ & -0.21 & -0.12 & -0.24 & -0.22 & 0.03 & -0.26 & -0.03 & -0.05 \\
C.S. $(=1)$ & 0.64 & 0.28 & 0.71 & 0.81 & -0.24 & 0.39 & 0.24 & 0.41 \\
\hline Items & $\mathrm{R}_{9}$ & $\mathrm{R}_{10}$ & $\mathrm{R}_{11}$ & $\mathrm{R}_{12}$ & $\mathrm{R}_{13}$ & $\mathrm{R}_{14}$ & $\mathrm{R}_{15}$ & \\
\hline C.S. $(=0)$ & -0.52 & -0.07 & 0.05 & 0.04 & -0.27 & 0.07 & -0.05 & \\
C.S. $(=1)$ & 0.72 & 0.54 & -0.34 & -0.12 & 0.52 & -0.21 & 0.73 & \\
\hline
\end{tabular}

various concerns, such as compensation for some kinds of traffic accidents, even if they are neighbors. In any case, this result shows that the mechanism of deciding the impression of MASS as a transportation means is complicated, and there may be a structure where factors as explanatory variables have relationships with each other. Therefore, in the future, clarifying the relationship between explanatory variables is presumably important as a method to understand the mechanism of young people's judgment for MASS.

Table 8 shows the discriminating accuracy of the model $Q_{5}$, Table 9 shows its category scores, and Figure 3 shows partial correlation coefficients and ranges of explanatory variables. Table 8 indicates that the accuracy of the discrimination was reduced by the fact that many examinees were not favorable to MASS, but considered it necessary as a public transportation service. In other words, the model of $\mathrm{Q}_{1}$ and $\mathrm{Q}_{2}$ had a pattern of explanatory variables in which the value of the objective variable was 0 , while the model of $\mathrm{Q}_{5}$ had many data in which the value of the objective variable was 1 .

The $\mathrm{Q}_{5}$ model is relatively easy to understand the mechanism unlike the model of $\mathrm{Q}_{4}$ because the sign of the category score is ideal direction except for some explanatory 


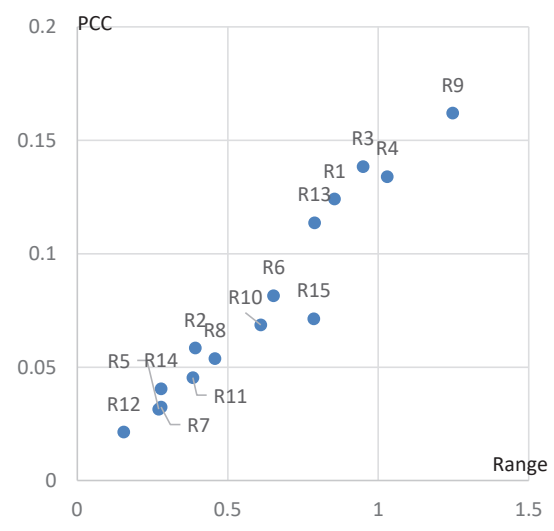

Figure 3: Partial correlation coefficients (PCC) and Range in the model $\mathrm{Q}_{5}$

variables. Even if the sign of the category score of the explanatory variables was not ideal, we don't care if its strength is not pronounced. We focus on three factors $R_{9}, R_{3}$, and $\mathrm{R}_{4}$ (see Figure 3 ) that are particularly strong. The interesting point is showing the strength of $\mathrm{R}_{4}$, "desire of contributing to the local community". Based on the hypothesis described in the previous section (many examinees were not favorable to MASS, but considered it necessary as a public transportation service), it can be read that there are local residents, including examinees who want to contribute to the community.

\section{Conclusion}

In this paper, to examine the sustainability of Mutual Assistance Support System (MASS), which is a Web service to mainly support vulnerable road users' daily life, we focused on the attitude survey result on young people's awareness about their resource sharing, including skills at their local community. Then, we applied Quantification Method Type II to analyze.

From the analysis result, we firstly showed the strong relationship between the question "When MASS is actually launched, do you want to use it as a user to provide your resource?" and its explanatory variables. Also, we confirmed the strong relationship between the question "When MASS is actually launched, do you want to use it as a user to get another one's resource?" and its explanatory variables. From this result, we concluded that young people's expectation for MASS strongly depends on the reasons or is summarized by the reasons prepared by this paper as the explanatory variables. The values of the category scores suggest that there is strong resistance to engaging with unknown people, even the local area familiar with the examinees. Fortunately, we could find that young people are willing to use MASS if they feel MASS convenient actively. On the other hand, some young people were reluctant to provide their resources because they are resistant to engaging with others. Participation by young people who can provide their resources is essential for the operation of MASS. Therefore, we understood the importance of giving some benefit that would be able to appeal to young people for MASS.

Next, from the analysis result using the answers on the social value of MASS, we could find the weak relationship between each examinee's subjective and objective views on the popularization of MASS. Concretely, there were a certain number of examinees thinking that MASS would be used in the area even they do not want to use it, and examiness of vice versa. From the analysis result, we found a possibility that even a person who was worried 
about some kinds of accidents/troubles or a person who was not interested in the local area, thought that a system not depending on public services and supporting mutual help is useful for supporting vulnerable road users and also local community.

While we were able to confirm the usefulness of Quantification Method Type II, we found its limitation. One analysis result suggested the importance for clarifying the relationship between explanatory variables. At this point, we assume that the mechanism of the reasons for deciding the impression of MASS is complicated, and there is a structure where factors as explanatory variables have relationships. Based on the assumption, as future work, we will apply Bayesian network to unveil the young people's attitude against MASS.

\section{Acknowledgment}

This work was partly spported by Japan Society for the Promotion of Science, Grant-in-Aid for Scientific Research (C), No.18K02214 and FIVE, Non-Profit Organization.

\section{References}

[1] Banai-Kashanai, A.R., Toward a Synthetic Measure of Good Settlement Form" Environment and Planning B, 15, pp.399-412 (1988).

[2] N. Ohigashi, A. Tanaka and S. Watanabe, Traffic Awareness Investigation by Questionary Survey in Housing Complexs, Research bulletin of the Hiroshima Institute of Technology, Vol.43, pp.113-117 (2009), In Japanese.

[3] N. Ohigashi and S. Matsumoto, Research on the Possibility of Riding-together Traffic in Residential Estates, Bulletin of Hiroshima Institute of Technology, Research, Vol.49, pp.19-22 (2015), In Japanese.

[4] S. Matsumoto and N. Ohigashi, Examining an Effective Way to Support Vulnerable Road Users in Itsukaichi District, Hiroshima City, Information Engineering Express, Special issue on Business Management of Technology, Vol.2, No.3, pp.43-52 (2016).

[5] S. Matsumoto, N. Ohigashi, T. Hasuike, Developing a Transportation Support System for Vulnerable Road Users in Local Community, Proc. of 2016 5th IIAI International Congress on Advanced Applied Informatics, pp.797-800 (2016).

[6] S. Matsumoto, N. Ohigashi and T. Hasuike, Design and Development of a Web Service to Support Daily Life of Vulnerable Road Users in Suburban Residential Estates in Hiroshima City, Proc. of 2017 6th IIAI International Congress on Advanced Applied Informatics, In USB (2017).

[7] S. Matsumoto, N. Ohigashi, T. Hasuike, Livelihood Assistance System for Vulnerable Road Users in Suburban Residential Areas based on Mutual Assistance, International Journal of Service and Knowledge Management, ISSN: 2189-9231, Vol 1, No 2, pp.13-31 (2017)

[8] D. Demailly, A. Novel, The sharing economy: make it sustainable. Studies, (03/14), 30 (2014). 
[9] J. Hamari, M. Sjoklint, A. Ukkonen, The sharing economy: Why people participate in collaborative consumption. Journal of the Association for Information Science and Technology, (2015).

[10] T. Dillahunt, A. Malone, The promise of the sharing economy among disadvantaged communities. In Proceedings of the 33rd Annual ACM Conference on Human Factors in Computing Systems, pp.2285-2294. ACM (2015).

[11] D. Rauch, D. Schleicher, Like uber, but for local government law: The future of local regulation of the sharing economy. Ohio St. LJ, 76, 901 (2015).

[12] D. Wosskow, Unlocking the sharing economy: An independent review (2014).

[13] S. Denning, An economy of access is opening for business: five strategies for success. Strategy \& Leadership, 42(4), pp.14-21 (2014).

[14] S. Matsumoto, N. Ohigashi, Attitude Survey of Young People to Examine the Usefulness of a Skill Sharing Web Service for Regional Vulnerable Road Users, Proceeding of 7th International Congress on Advanced Applied Informatics, pp.724-729 (2018).

[15] P. White, Public transport: its planning, management and operation. Taylor \& Francis (2016).

[16] C. Mulley, J. Nelson, Flexible transport services: A new market opportunity for public transport. Research in Transportation Economics, 25(1), pp.39-45 (2009).

[17] C. Mulley, J. Nelson, R. Teal, S. Wright, R. Daniels, Barriers to implementing flexible transport services: An international comparison of the experiences in Australia, Europe and USA. Research in Transportation Business \& Management, 3, pp.3-11 (2012).

[18] Y. Kawamoto, Car Corporative and Car Sharing Feasibility in the aged and Depopulated Village, Research report of Grant-in-Aid for Young Scientists (B), No.21760399 (2009-2010), In Japanese.

[19] M. Kawabata, S. Matsumoto, K. Sano, S. Tsuchiya, Car Passenger Transport and Barriers to Community Bus Use in a Mountainous Underpopulated Region in Japan. Journal of the Eastern Asia Society for Transportation Studies, 8, pp.497-510 (2010).

[20] T. Tsuboyama, K. Mizuno, H. Sasaki and S. Nishihara, Development of a simulation environment for dial-a-ride systems using swarm, Proceedings of the 75th National Convention of Information Processing Society of Japan, pp.129-131 (2013), In Japanese.

[21] K. Tsubouchi, H. Yamato and K. Hiekata, Development of the Simulator for the Ondemand Bus Introduction, The Japanese Society for Artificial Intelligence, Vol.25(3), pp.400-403 (2010), In Japanese.

[22] A. Kleiner, B. Nebel, V. Ziparo, A mechanism for dynamic ride sharing based on parallel auctions, Proc. of 22th International Joint Conference on Artificial Intelligence, pp.266-272 (2011). 
[23] N. Agatz, A. Erera, M. Savelsbergh, X. Wang, Optimization for dynamic ride-sharing: IIIAA review, European Journal of Operational Research, Vol.223(2), pp.295-303 (2012).

[24] H. Yotsutsuji, K. Sasaki, M. Yamamoto, Availability of volunteer-based dynamic ridesharing with bipartite group in a low-density small community in Japan. Journal of the Eastern Asia Society for Transportation Studies, 10, pp.1009-1024 (2013).

[25] Y. Takamura, Ways of involvement of the government and the citizens towards solving the problems associated with the housing complexes in the suburb, Bulletin of the Faculty of Urban Management, Fukuyama City University, Vol.2, pp.33-50 (2013), In Japanese.

[26] S. Matsumoto, N. Ohigashi and S. Abe, An equipment to support transportation of people and luggages in daily life, Japanese Patent Application No. 2015-160396 (2015), In Japanese. 